\title{
Scale Effects in the Steering Time Difference between Narrowing and Widening Linear Tunnels
}

\author{
Shota Yamanaka \\ Meiji University and JSPS \\ Nakano, Tokyo, Japan \\ stymnk@meiji.ac.jp
}

\author{
Homei Miyashita \\ Meiji University \\ Nakano, Tokyo, Japan \\ homei@homei.com
}

\begin{abstract}
Steering time differs between narrowing and widening linear tunnels; a narrowing tunnel requires more time to navigate than a widening one. A prediction model, $I D_{\mathrm{Gap}}$, for the time difference has recently been proposed, and it shows an excellent fit. However, the time difference in movement and model fitness were confirmed on a limited scale. The experiment used a 13.3-inch pen tablet, which required primarily wrist movements with a particular level of forearm extension. In this study, we tested the scale effects in the steering time difference between the two tunnel types. In our experiment, participants performed steering operations at five scales, from the entire 21.5-inch tablet area to its 1/12-scale size. The results always showed the time difference, and the conventional steering law did not show a good fit. ID $D_{\text {Gap }}$ improved the fitness, thereby confirming the validity of the model. The scale effects for the other results, including error rates and index of performance, are also discussed.
\end{abstract}

\section{Author Keywords}

Scale effects; motor scale; visual scale; steering law; graphical user interface; human performance.

\section{ACM Classification Keywords}

H.5.2. Information interfaces and presentation (e.g., HCI): User Interfaces.

\section{INTRODUCTION}

Modeling human performance is an important theme in human-computer interaction (HCI). There have been many studies on the derivation of robust models and the correction thereof to adapt to various situations. Fitts' law [9], a well-known model in HCI especially for graphical user interfaces (GUIs), expresses the relationship between the target pointing task's difficulty and its movement time $(M T)$. Another well-known robust model is the steering law [1], which models complicated tasks such as drawing,

\footnotetext{
Permission to make digital or hard copies of part or all of this work for personal or classroom use is granted without fee provided that copies are not made or distributed for profit or commercial advantage and that copies bear this notice and the full citation on the first page. Copyrights for thirdparty components of this work must be honored. For all other uses, contact the Owner/Author.

Copyright is held by the owner/author(s).

NordiCHI '16, October 23-27, 2016, Gothenburg, Sweden

ACM 978-1-4503-4763-1/16/10.

http://dx.doi.org/10.1145/2971485.2971486
}

writing, and in particular, navigating a path.

We use various sizes of displays and input devices depending on situations; therefore, the required operation granularity varies. We are often forced to move the entire upper limb in a wide range of movements, and in some cases, only finger movements are required-e.g., drawing with a large liquid crystal pen tablet and taking notes on a smartphone, respectively. There have been several studies on human performance being influenced by such scale effects. According to Accot and Zhai's steering task experiment [3], the best input size of a pen tablet was A5, which required wrist movements with the extension of the forearm, with rather small or large sizes degrading the performance in terms of $M T$ or error rate. However, the steering law held in any input area scaling from $28 \times 19$ $\mathrm{mm}$ to $455 \times 303 \mathrm{~mm}$. In contrast, pointing tasks have a different result; Fitts' law shows a bad fit when pointing at a very small target (1-8 pixels) [7]. This indicates that the effectiveness of performance models decreases in some scale conditions and the scaling effect is a factor in a model's validity. If we were to use a performance model without testing scale effects, we would obtain a false estimate of task difficulty or predict an incorrect $M T$ and we would discuss the effectiveness of novel interaction techniques based on incorrect calculations; this poses a serious problem for the future of $\mathrm{HCI} / \mathrm{GUI}$ research.

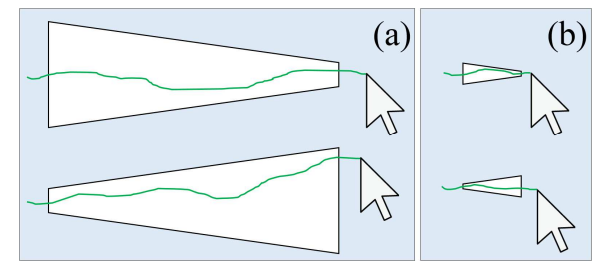

Figure 1. Scale of (a) is five times that of (b)-e.g., 30 and $6 \mathrm{~cm}$ in the tunnel lengths, respectively - but they have the same value of steering law difficulty. On any scale, is there always a steering time difference between narrowing and widening tunnels?

In this study, using a direct-input stylus, we discuss the scale effects in the steering time difference between narrowing and widening linear tunnels. Quite recently, Yamanaka and Miyashita found that $M T$ for a narrowing tunnel $\left(M T_{\mathrm{NT}}\right)$ is significantly greater than $M T$ for a widening tunnel $\left(M T_{\mathrm{WT}}\right)$, and they derived a difficulty difference model called $I D_{\mathrm{Gap}}$ [24]. The validity of this model was confirmed by an experiment using tunnels of 
amplitude $A$ between 61.2 and $122.4 \mathrm{~mm}$ and width $W$ between 2.2 and $10.4 \mathrm{~mm}$ requiring primarily wrist movements with a particular level of forearm extension. However, considering scale effects, we have several concerns regarding their study, which are as follows:

- At a very small scale, passing through a widening tunnel becomes more difficult; therefore, $M T_{\mathrm{WT}}$ increases and its difference from $M T_{\mathrm{NT}}$ decreases.

- At a very large scale, passing through a narrowing tunnel becomes less difficult; therefore, $M T_{\mathrm{NT}}$ decreases and its difference from $M T_{\mathrm{WT}}$ decreases.

If there is no large difference between $M T_{\mathrm{NT}}$ and $M T_{\mathrm{WT}}$ i.e., if the merged $M T$ of $M T_{\mathrm{NT}}$ and $M T_{\mathrm{WT}}$ shows a good fit with the conventional steering law-using the $I D_{\text {Gap }}$ model is unnecessary because $M T$ can be predicted irrespective of the tunnel type (narrowing or widening). In other words, is the $I D_{\text {Gap }}$ model required in a particular range of scale conditions, or is it required regardless of the scale (see Figure 1)? To answer these questions, we conduct an experiment involving steering tasks with scale changes.

\section{RELATED WORK}

\section{Steering Law}

The performance of passing through straight and circular tunnels of length $A$ and constant width $W$ is modeled by the steering law [1]:

$$
M T=a+b \times \frac{A}{W}
$$

where $a$ and $b$ are empirically determined constants and $A / W$ is the index of difficulty $(I D)$. Equation 1 means that, for example, a longer or narrower path has a higher $I D$ and navigating that path requires a longer $M T$. This law was first confirmed using an indirect-input stylus [1] and later using a mouse, trackball, touchpad, and pointing stick [2]. The steering law showed a good fit with various conditions such as changes in cursor size [20], degree of surface friction on a stylus pad [23], motor scale (control-display (CD) gain) [3], and start position [26], as well as the addition of a fisheye-view effect [13]. In addition to twodimensional GUI operations, the applicability of the steering law has been demonstrated in passing through a three-dimensional (3D) pipe [5, 19] and driving a car [27].

Several revisions of the steering law have been proposed. Accot and Zhai derived the $I D_{\mathrm{NT}}$ model to navigate a narrowing, straight tunnel (as illustrated in Figure 1) [1]:

$$
I D_{\mathrm{NT}}=\frac{A}{W_{R}-W_{L}} \ln \frac{W_{R}}{W_{L}}
$$

where $W_{L}$ and $W_{R}$ are the left- and right-end widths of the tunnel, respectively. Other revised models are for the hovering operation with a stylus [16], maintaining pressure within a specified level using a stylus [25], passing through a tunnel with corners [21], and pointing to an item at the end of a path [18].
Equation 2 was derived as the integral of infinitesimal constant-width tunnels. However, Yamanaka and Miyashita indicated that the $I D$ for the opposite direction could be calculated using the same formula of Equation 2 [24]. Because the actual $M T_{\mathrm{NT}}$ was greater than $M T_{\mathrm{WT}}$, they proposed a corrected model that expresses the $I D$ difference, $I D_{\mathrm{Gap}}$, between narrowing and widening straight tunnels:

$$
I D_{\mathrm{Gap}(k)}=\frac{A\left(W_{L}-W_{R}\right)}{k W_{L} W_{R}}
$$

where $k$ is an empirically determined constant. They supposed that the $I D$ for a widening tunnel $\left(I D_{\mathrm{WT}}\right)$ was less than $I D_{\mathrm{NT}}$ by $I D_{\mathrm{Gap}}$. Their experimental results showed that passing through a narrowing tunnel was significantly more difficult than passing through a widening tunnel $\left(M T_{\mathrm{NT}}>\right.$ $\left.M T_{\mathrm{WT}}\right)$, and that the $I D_{\mathrm{Gap}}$ model showed a good fit $\left(R^{2}\right.$ $>$.99) when $k=3.14$. Note that their user study was conducted with a direct-input stylus; $A$ was 61.2 to 122.4 $\mathrm{mm}$, and $W$ (both $W_{L}$ and $W_{R}$ ) was 2.2 to $10.4 \mathrm{~mm}$. As mentioned in their report, the sizes of $A, W$, the display, and the input area of the tablet were limitations of their study. Tasks with these sizes can be performed primarily by wrist movements with the forearm extended. We have no idea whether there is a steering time difference among tunnel types on very small or large scales, e.g., a smartwatch display or a liquid crystal pen tablet for professional illustrators, respectively.

\section{Scale Effects}

Most previous work on the scale effect has studied pointing task performance. For a rich review of the literature, we refer readers to [7], which describes several definitions of the scale effect and resolution of input/output devices. To summarize, and in relation to our study, there are three scale effects:

- Visual scale: e.g., changing the display magnification ratio using a loupe tool.

- Motor scale: e.g., changing the mouse cursor speed (CD gain) via the operating system setting.

- Both visual and motor scales: e.g., zooming in to a map, which is visually magnified and for which cursor movements on the display increase.

In pointing tasks, both visual and motor scales affect the fitness of Fitts' law when using a mouse [7]. In particular, when the target size is very small (1-8 pixels), Fitts' law shows a bad fit. Another study showed that the Fitts' law fitness decreased when the screen size was very small (3.9 inches) [4]. Even with a robust Fitts' law, scale effects could reduce the model's fitness.

Scale effects in the performance of pointing and pointinglike tasks have also been studied in terms of CD gain. One of the characteristics of human motor performance is an inverted U-shaped function, wherein the performance approaches the highest point at the medium range of the motor scale [14]. In simple terms, human hands cannot 
comfortably perform actions that require an excessively wide range or excessively fine adjustment, and there is a most suitable scale between those. This function was confirmed using an isometric joystick (rate control) by Hess [14]. However, Gibbs suggested a linear function of scale effects: a smaller motor scaling reduces the targeting performance in both rate and position control, where there is no system lag [11]. In addition, the absence of an effect of the motor scale in mouse pointing tasks was described as follows: "... changing the gain does not speed mouse performance" [15].

In contrast to pointing task performance, there have been few studies on scale effects in steering task performance. Accot and Zhai investigated the motor scale effect using an indirect stylus, which eliminated the effect of the visual scale [3]. They controlled the tablet-pad scale from the entire $1 / 1$ size $(455 \times 303 \mathrm{~mm})$ to $1 / 16$ size $(28 \times 19 \mathrm{~mm})$, with fixed displayed tunnel sizes. The results showed that the steering law held on any motor scales, but excessively large $(1 / 1)$ or small $(1 / 8$ and $1 / 16)$ scales significantly degraded the performance. Their results suggested that there is a U-shaped function in steering operations. Senanayake and Goonetilleke conducted a steering task experiment using an optical mouse with a $\mathrm{CD}$ gain of 2.3, 10 , and 15 [22]. The result showed a U-shaped function; a medium gain was the best for mouse steering.

Assuming that scaling is a factor in steering performance and that there is a U-shaped function, we have a concern regarding whether the $I D_{\text {Gap }}$ model holds at any scale. The previous study [24] showed a good fit for the model, but the scale of the experiment might be suitable for path steering. Also, there was a significant difference between $M T_{\mathrm{NT}}$ and $M T_{\mathrm{WT}}$. It is unclear that this tendency would be observed at other scales, such as a tunnel with a 1 or $2 \mathrm{~cm}$ amplitude and $1 \mathrm{~mm}$ width; consequently, we conduct an experiment with various scales.

\section{EXPERIMENT}

In this experiment, participants performed steering in narrowing and widening linear tunnels at five different scales using a direct-input pen tablet. The selected scales covered a wide range of movements from those requiring only fingertip movements to those requiring entire arm movements. We wanted to investigate the necessity or limitations of the $I D_{\text {Gap }}$ model in scaling - whether $I D_{\text {Gap }}$ is valid only at a limited range of scales of wrist movements or also in a wider range.

Note that our experiment differs somewhat from Accot and Zhai's scale effect study [3]. First, we use a direct-input method, which changes both visual and motor scales. Liquid crystal pen tablets are easily available and provide a common environment for drawing applications. In addition, the previous study on the steering time difference between tunnel types was conducted with a direct-input stylus [24]; consequently, we use that input method here as well. We will discuss the experimental design for the case of an indirect-input stylus, which can separate visual and motor scales. Second, although we also discuss the performance differences among different scales, our primary interest is in the performance difference between narrowing and widening tunnels at the same scale. We would like to test whether there is always a steering time difference at any scale.

\section{Participants}

The participants were ten graduate and undergraduate university students (two females, eight males, average age = $22.1, S D=1.87$ years). All participants had normal or corrected-to-normal vision. All participants were righthanded and operated the stylus with the right hand. One participant had approximately two years of experience in habitually using a stylus with his smartphone to take notes, and another had approximately seven years of previous experience with an indirect-input stylus.

\section{Apparatus}

We used a desktop PC (Core i7-3930K, $3.2 \mathrm{GHz} \times 6$ cores, 16 GB of RAM, Windows 7 Pro) with an NVIDIA GeForce GTX 660 graphics card. The display and input device was an IPS liquid crystal pen tablet, Wacom Cintiq 22HD $(475.2 \times 267.3 \mathrm{~mm}$ active input area, $1920 \times 1080$ pixels resolution, $4.04 \times 4.04$ pixels $/ \mathrm{mm}$ density, 21.5 inches in diameter, $0.005 \mathrm{~mm}$ sensitivity resolution). The experimental system implemented with Hot Soup Processor 3.4 was displayed in full-screen mode. The system runs at approximately $125 \mathrm{~Hz}$.

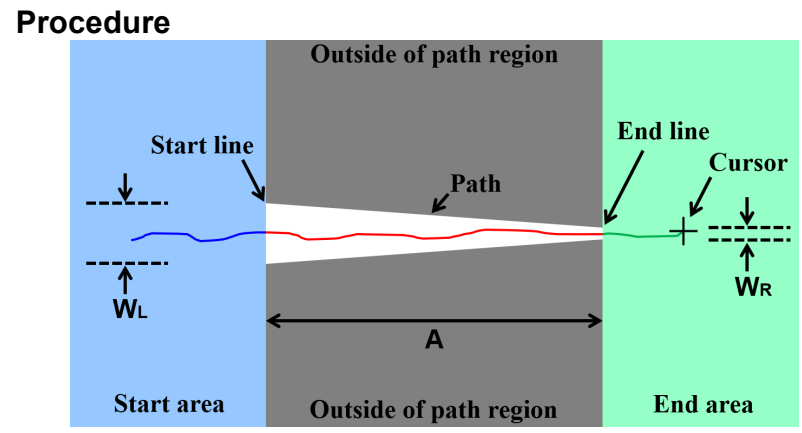

Figure 2. Screen layout of the experiment for the case of a narrowing tunnel.

When the task started, a screen with several colors was displayed on a liquid crystal pen tablet, as shown in Figure 2. The participant touched the stylus to the start area and then navigated past the start line from left to right. The participant then navigated the stylus tip all the way past the end line, all while attempting to stay within the bounds of the path. Removing the stylus from the end area then displayed a screen, which showed the next selection of parameters. Participants were instructed to perform the required operation as quickly and accurately as possible.

When the stylus was brought close to the tablet surface, a black crosshair cursor 25 pixels $(6.19 \mathrm{~mm})$ in length was displayed. The cursor trace left a blue line when it was in 
the start area. Upon crossing the start line, the cursor trace turned red. Passing the end line triggered a bell to signal a successful attempt and prompted the cursor trace in the end area to turn green. The cursor trace turned black when it was moved outside the path.

When the cursor moved outside the path or the stylus left the tablet surface, a beep sounded, and the attempt was suspended. The participant set down the stylus, lifted it again, and then re-entered the start area to start the test again. However, in this experiment, only the case wherein the cursor moved outside the path was recorded as an error. The stylus leaving the surface was regarded as a different type of error than that which was the focus of the current experiment (i.e., the effect of the change in width and scale effects) and was thus not regarded as an error. These are the same conditions as those of previous studies [3, 24]. Note that after passing through a tunnel, going to the outside area of the task-drawn region-i.e., the blackout area on scales $S$ $=2$ to 12 (see Figure 3 ) is not prohibited and not regarded as an error. This is also the case for $S=1$; the pen tip can enter the bezel from the active input area without a physical step. The steering law considers errors only in the actual tunnel; therefore, the participants' actions after the conclusion of a task trial are ignored. If participants must stop the pen tip in a specific area after the goal line, it is called a targeted-steering task, which has another model formula $[8,18]$.

\section{Design}

In our experiments with scale $S=1$ (using the entire display size, details below), the distance $A$ was 600 and 1560 pixels (148.5 and $386.1 \mathrm{~mm}$ ). The path width $W$ for both the left start line $\left(W_{L}\right)$ and the right end line $\left(W_{R}\right)$ was 36,108 , and 180 pixels $(8.9,26.7$, and $44.6 \mathrm{~mm})$, for which only combinations in which $W_{L} \neq W_{R}$ could be selected. The tunnel type (narrowing or widening) was determined according to the combination of $W_{L}$ and $W_{R}$.

The total number of parameter combinations was $2(A) \times(3$ $\left(W_{L}\right) \times 3\left(W_{R}\right)-3$ (excluding $\left.\left.W_{L}=W_{R}\right)\right)=12$ conditions, with an $I D$ range between 4.26 and 23.8. Each $S$ condition included five blocks of the 12 randomly ordered conditions, with the first block being a practice block and the remaining four blocks comprising the actual tasks. The data recorded for the actual tasks totaled 12 conditions $\times 4$ blocks $\times 5$ scales $\times 10$ participants $=2400$ trials. The order of the five scales was balanced among five groups of participants using a Latin square pattern.

Before the actual experiment, participants adjusted the height of the seat. During the practice block in each $S$ condition, the angle and position of the tablet on the table could also be adjusted. The total time taken was approximately 25 minutes, from the preliminary instructions to the completion of all tasks. After completion, participants completed a questionnaire concerning their impressions of the experiment.
Scale (S) Conditions

We chose five scales for the participants to perform with different strategies of joints. Figure 3 illustrates the relative sizes of active areas on the tablet for the different movement scales. The ratio of the display and input area was determined by $1 / S$, if $S=1$, the entire active area of the tablet was used. Table 1 shows the path widths and distances for each scale condition in the physical unit ( $\mathrm{mm}$ ). The scales used were as follows:

- Very large scale $(S=1)$ : the entire active area of the tablet $(475.2 \times 267.3 \mathrm{~mm})$ was used. Participants were required to move the entire arm and hand using the shoulder, elbow, and wrist joints.

- Large scale $(S=2): 1 / 2$ of the active area of the tablet $(237.6 \times 133.7 \mathrm{~mm})$ was used. This size is slightly larger than the display sizes of a 10.1 -inch wide monitor tablet $(223.6 \times 125.8 \mathrm{~mm})$ or iPad $(197.0 \times 147.8 \mathrm{~mm})$. Participants primarily move the forearm, but they might be required to move the upper arm to perform the tasks quickly.

- Middle scale $(S=4): 1 / 4$ of the active area of the tablet $(118.8 \times 66.8 \mathrm{~mm})$ was used. This is slightly smaller than a 5-inch wide smartphone display $(110.7 \times 66.3 \mathrm{~mm})$ such as the Sony Xperia Z, which requires primarily wrist movements with an extension of the forearm. In our five $S$ conditions, this is the scale most similar to that in the study by Yamanaka and Miyashita ( $A$ ranged from 61.2 to $122.4 \mathrm{~mm})[24]$.

- Small scale $(S=9): 1 / 9$ of the active area of the tablet $(52.8 \times 29.7 \mathrm{~mm})$ was used. This is smaller than the touch-display size of the Nintendo 3DS $(61.4 \times 46.1 \mathrm{~mm})$, and it might be smaller than that of the participants' daily touchscreen usage with a stylus. This scale requires primarily movement of the fingers and wrist.

- Very small scale $(S=12): 1 / 12$ of the active area of the tablet $(39.6 \times 22.3 \mathrm{~mm})$ was used. This size resembles fitness bands or smartwatch displays, e.g., smaller than the Samsung Gear Fit $(44.8 \times 13.3 \mathrm{~mm})$ and larger than the LG-W100 $(29.6 \times 29.6 \mathrm{~mm})$. Participants are required to move the wrist and fingers very carefully with the shoulder and elbow joints stabilized. Note that the smallest $W$ of $0.7 \mathrm{~mm}$ is far above the tablet's sensitivity; hence, the system can track the pen tip with sufficient accuracy.

\section{Collected Data}

The time $M T$ taken to pass the end line after crossing the start line, the error rate, and a time-stamped cursor trace were all recorded. Further, the cursor speed along the x-axis and the standard deviation $S D_{\mathrm{y}}$ of the movement along the $y$-axis were calculated using the cursor trajectory data. These data were collected only in the actual tunnel, depicted as the red trajectory (Figure 2). The analysis method of effective parameters [17] was not used in this study. 

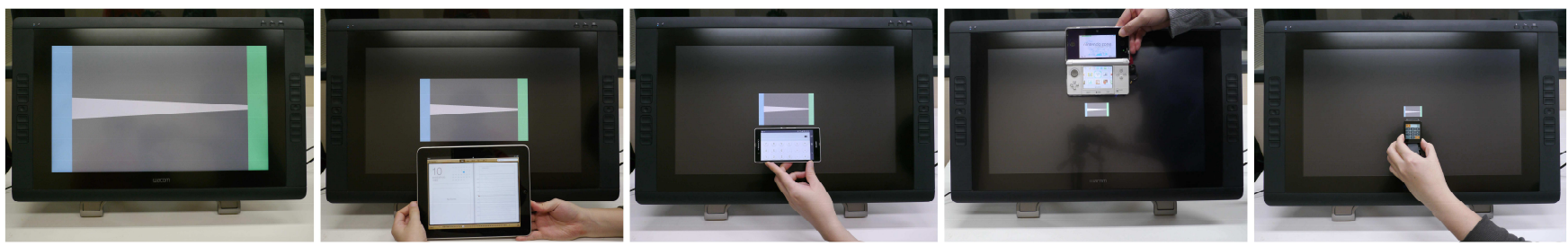

Figure 3. Relative active sizes of the tablet at different scales. (left to right) $S=1, S=2$ with iPad, $S=4$ with Xperia $Z, S=9$ with Nintendo 3DS, and $S=12$ with LG-W100.

\begin{tabular}{l|r|r|r|r|r}
$\begin{array}{l}W \text { and } A \\
\text { [pixels] }\end{array}$ & $S=1$ & $S=2$ & $S=4$ & $S=9$ & $S=12$ \\
\hline$W=36$ & 8.9 & 4.5 & 2.2 & 1.0 & 0.7 \\
\hline$W=108$ & 26.7 & 13.4 & 6.7 & 3.0 & 2.2 \\
\hline$W=180$ & 44.6 & 22.3 & 11.1 & 5.0 & 3.7 \\
\hline$A=600$ & 148.5 & 74.3 & 37.1 & 16.5 & 12.4 \\
\hline$A=1560$ & 386.1 & 193.1 & 96.5 & 42.9 & 32.2
\end{tabular}

Table 1. Movement widths and distances in input space for each scale condition (unit: $\mathrm{mm}$ ).

\section{Results}

The dependent valuables were $M T$, the error rate, the cursor speed, and the standard deviation $S D_{\mathrm{y}}$. In total, 2536 trials were performed. After excluding 136 error trials, we analyzed the data via repeated measures, using ANOVA and the Bonferroni post hoc test. The independent variables were scale $S$, tunnel type, and $I D$.

\section{Steering Time}

Figure 4 shows the average $M T$ for both tunnel types at each scale, and Figure 5 shows the index of performance $(I P)$ at each scale. We observed the main effects of $S\left(F_{4,36}\right.$ $=6.632, p<.001)$, tunnel type $\left(F_{1,9}=15.664, p<.01\right)$, and ID $\left(F_{5}, 45=29.756, p<.001\right)$ on MT. The post hoc test shows that navigation is significantly slower with $S=12$ than with $S=9(p<.05)$, and widening is passed faster than narrowing $(p<.01)$, for which the average times were $M T_{\mathrm{WT}}=585 \mathrm{~ms}$ and $M T_{\mathrm{NT}}=821 \mathrm{~ms}$. We also observed significant interaction between scale and tunnel type $\left(F_{4,36}\right.$ $=2.750, p<.05)$. Small scales $(S=9$ and 12) appear to have required more steering time, but paired tests between scale levels show no significant effect for both tunnel types (all $p>.05$ ).

The top row of Figure 6 shows the relationship between $I D$ and $M T$ for both tunnel types at each scale. We observe that the steering law held with good regression fitness at all scales $\left(R^{2}>.94\right)$, showing that our experimental settings (e.g., ranges of scale, $I D$, physical amplitude, or physical width) were of no consequence as steering law tasks. One might notice that $M T$ tended to be longer (= steeper slope) at small scales, particularly $S=12$ in the top row of Figure 6 . This means that the participants performed less well in smaller tunnels. To confirm this, we compared $I P$ values, defined as $1 / b[\mathrm{bits} / \mathrm{s}]$, at each scale. We observed the main effects of scale $\left(F_{4,36}=3.664, p<.05\right)$ and tunnel type $\left(F_{1,9}\right.$ $=25.159, p<.01)$ on $I P$. The post hoc test shows that the performance was significantly lower with $S=12$ than with $S=1$ or $9(p<.05$ for both), and narrowing is worse than widening $(p<.05)$. There is no significant interaction between scale and tunnel type $(p=.398)$.

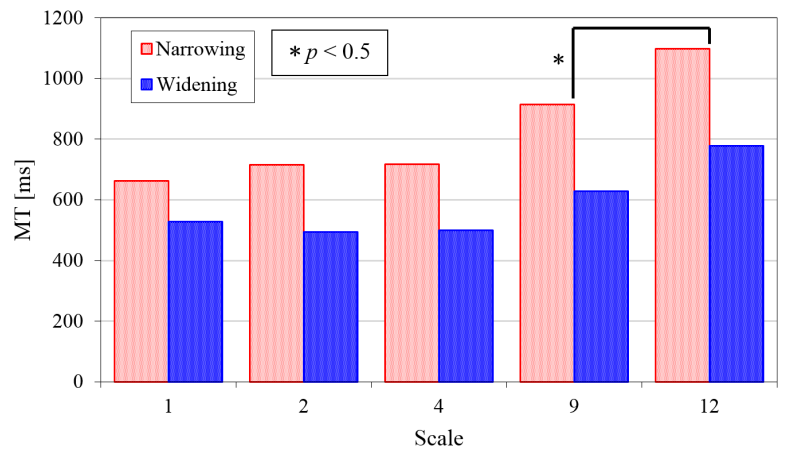

Figure 4. Average movement times at each scale divided by the tunnel type.

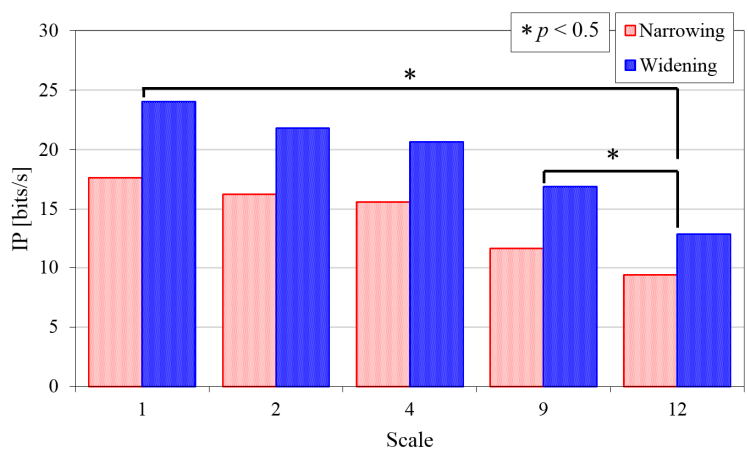

Figure 5. Index of performance at each scale divided by the tunnel type.

Speed

Figure 7 shows the average speed profiles in the tunnels. We observed the main effects of $S\left(F_{4,36}=77.151, p\right.$ $<.001)$, tunnel type $\left(F_{1,9}=43.332, p<.001\right)$, and $I D\left(F_{5,45}\right.$ $=95.373, p<.001)$. The post hoc test shows that the larger the scale is, the higher the speed the participants reach ( $p$ $<.01$ at least for all pairs). In widening, the speed is higher than in narrowing $(p<.001)$. The average speeds for the narrowing and widening directions were 0.879 pixels $/ \mathrm{ms}$ $(0.218 \mathrm{~mm} / \mathrm{ms})$ and 1.041 pixels $/ \mathrm{ms}(0.258 \mathrm{~mm} / \mathrm{ms})$, respectively. 

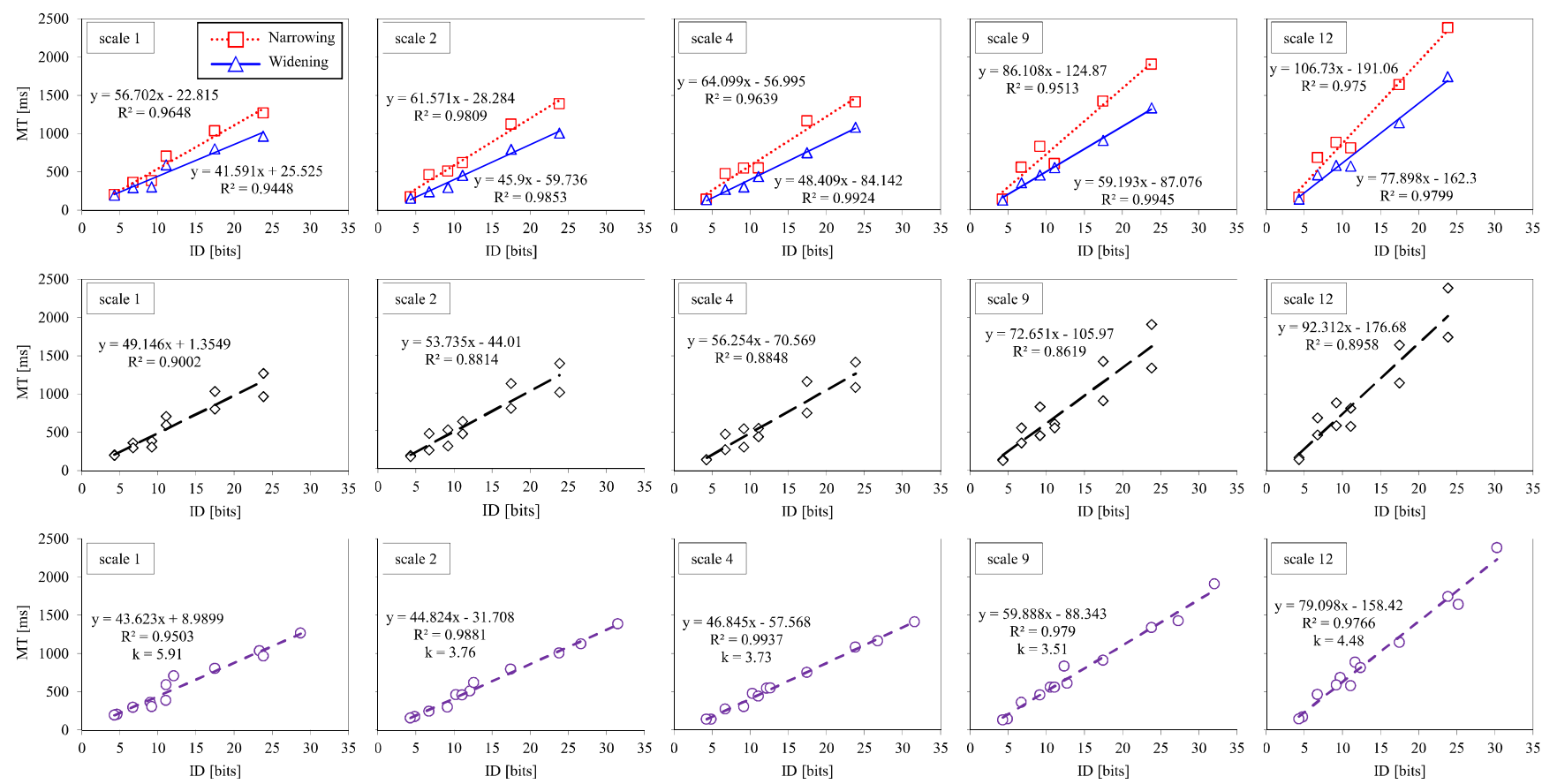

Figure 6. Movement time versus index of difficulty. (top row) The steering law of Accot and Zhai's model [1] shows good fits where the tunnel types are divided, as suggested by Yamanaka and Miyashita [24]. (middle row) Without type division, the steering law does not show good fits, which means that one regression expression cannot predict the movement time accurately. (bottom row) IDGap model [24] shows good fits using one regression expression where the tunnel types are not divided.
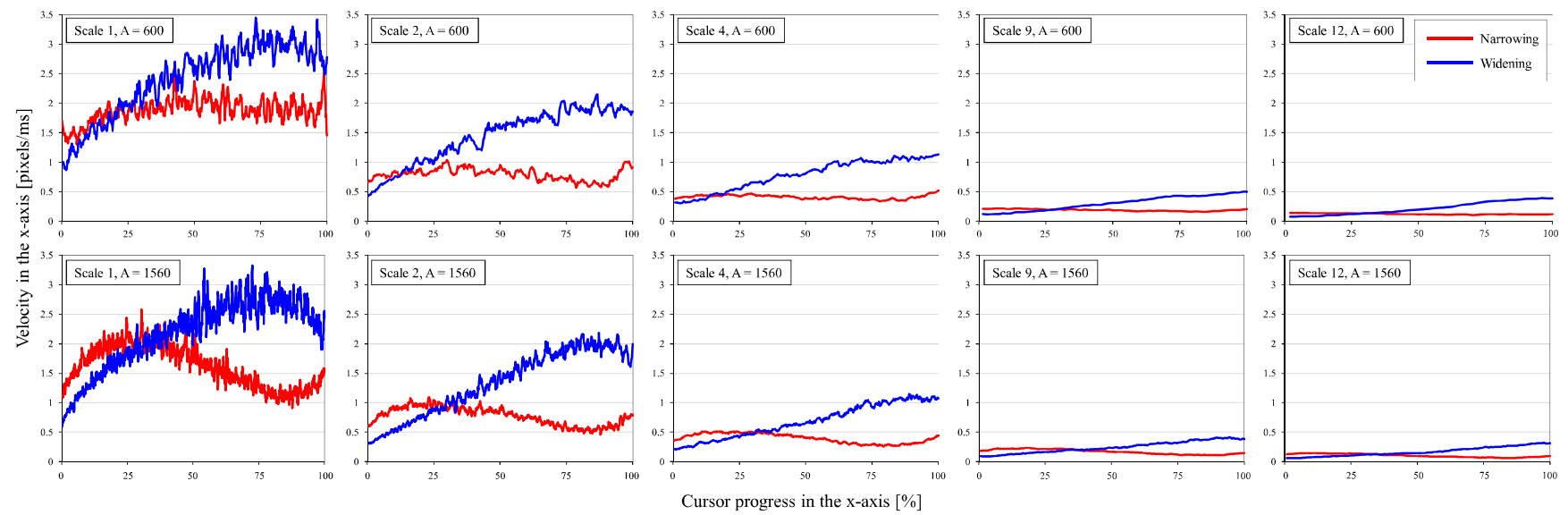

Figure 7. Average speed profiles at each scale and each amplitude filtered by a seven-point simple moving average.

\section{Errors}

Figure 8 shows the average error rates for both tunnel types at each scale. We observed the main effects of $S\left(F_{4,36}=\right.$ $11.289, p<.001)$, tunnel type $\left(F_{1,9}=7.579, p<.05\right)$, and ID $\left(F_{5,45}=9.151, p<.001\right)$. The post hoc test shows that more errors occur with $S=12$ than with $S=2(p<.05)$, and narrowing produces more errors than widening $(p<.05)$. The average error rates for the narrowing and widening directions were $7.48 \%(97 / 1297)$ and $3.15 \%(39 / 1239)$, respectively. There was no significant interaction between scale and tunnel types $(p=.141)$.

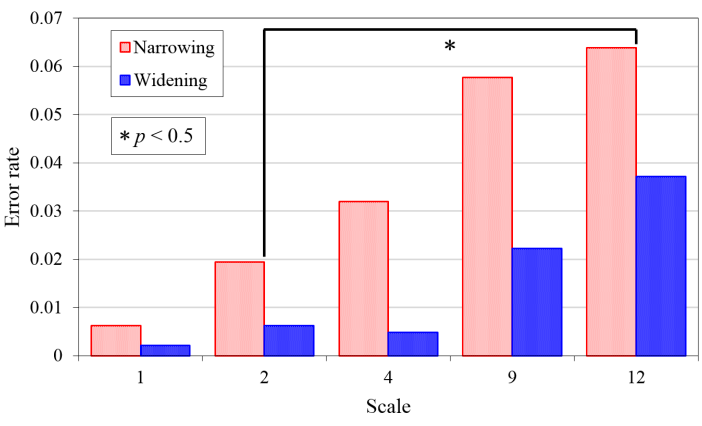

Figure 8. Average error rates at each scale divided by the tunnel type. 
Standard Deviation along Y-axis $\left(S D_{y}\right)$

We observed the main effects of $S\left(F_{4,36}=51.576, p\right.$ $<.001)$ and $I D\left(F_{5,45}=10.004, p<.001\right)$. We observed no significant effect of tunnel type $(p=.054)$. The post hoc test shows that $S D_{\mathrm{y}}$ increases with increasing scale (from $p$ $<.001$ to .01$)$, except for $S=9$ and $S=12(p=.378)$. The average $S D_{\mathrm{y}}$ values for the narrowing and widening directions were 2.91 pixels $(0.720 \mathrm{~mm})$ and 3.32 pixels $(0.821 \mathrm{~mm})$, respectively.

\section{DISCUSSION}

\section{Steering Time and Errors}

Interestingly, in contrast to Accot and Zhai's study, there appears to be no U-shaped function in our results. This means that scales primarily requiring wrist movements were not the best for users in our experimental conditions. $I P$ values in our study ranged from approximately 10 to 25 bits/s and were greater than Accot and Zhai's constantwidth linear tunnel results (range: 11 to $16 \mathrm{bits} / \mathrm{s}$ ) [3]. In addition, the error rate of the narrowing tunnel $(7.48 \%)$ was less than Accot and Zhai's narrowing tunnel result (18\%) [1]. The greater $I P$ and the lower error rate in our results indicate that our experimental condition was easier than that of the previous studies $[1,3]$, but we need to identify the reason for this.

One possible reason would be the input device; we used a direct-input stylus, whereas Accot and Zhai used an indirect type. For crossing tasks, a direct-input stylus is significantly superior to an indirect one in terms of movement time [10]. Another reason was the task parameters; $W$ values in our experiment were large, resulting in tunnel shapes resembling ballistics, and the participants were able to pass through in a short time. In our experiment, the smallest $W$ at the smallest scale $(S=12)$ was 3 pixels $(0.7 \mathrm{~mm})$. For even smaller $W$ values, i.e., 1 or 2 pixels, we had concerns that participants would make more errors and thus introduce "noise" into our experimental goal, i.e., scale effects. Next, we chose two further $W$ values based on the smallest $W$; these were 9 and 15 pixels $(2.2$ and $3.7 \mathrm{~mm}$ ) at $S=12$. This was in reference to Yamanaka and Miyashita's condition ( $W=11,31$, and 51 pixels; approximately 1,3 , and 5 times the smallest $W$ ) [24]. If we chose smaller $W$ values based on the smallest $W$, e.g., $W=3,4$, and 5 pixels, these would reduce the difference between $W_{L}$ and $W_{R}$, with the result that the tunnels would closely resemble a constant-width straight shape. Because our goal was to investigate scale effects in the steering time difference between narrowing and widening tunnels, very close values of $W$ must be rejected. Based on the chosen $W$ values (3, 9, and 15 pixels) at $S=12$, it was unavoidable that the tunnel width became easy to pass through at large scales (e.g., $W=36,108$, and 180 pixels $(8.9,26.7$, and $44.6 \mathrm{~mm})$ at $S=1)$, and the $I P$ values would become high. If other task parameter designs are chosen, for example, $A=1560$ pixels, $W_{L}=11$ pixels, and $W_{R}=31$ pixels, we will observe results different from those of our current experiment.
Assuming that the U-shaped function can be observed at a particular level of difficulty, perhaps our experimental setting might cover less than half of Accot and Zhai's result [3]. In other words, only one side of the U-shaped function in the observation that "the performance decreased as the scale shrank" might have been observed in our study. In this study, because our primary interest was not to check the U-shaped function across different scales, we did not tune the parameters to provide the U-shaped performance function. If someone would like to conduct a user study to check such functions, we suggest that more difficult tasks, particularly smaller $W$ values, be chosen.

Our results provide an operational implication; zooming in is better than as-is in drawing. For example, one who draws or paints a part of a width-changing path, such as that shown in Figure 9, must first zoom in to the illustration. Zooming in-i.e., operating at a larger scale for both motor and visual components - allows reduction in errors (going out of the hair boundary) without lengthening the drawing time. Obviously, a zooming operation itself requires a specific amount of time, but the illustrator can cut off the number of painting mistakes; hence, the number of undo/redo operations will also decrease.
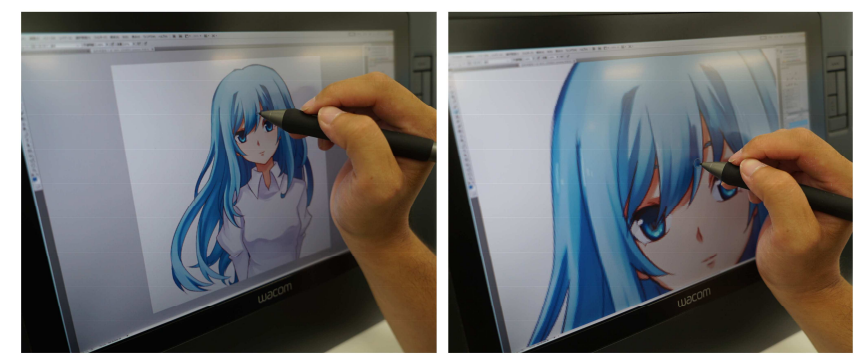

Figure 9. Drawer strokes a shadowing line while avoiding running over the hair boundary. Drawing (left) without and (right) after zooming in. () Yuka Shimaya

\section{Speed}

As shown in Figure 7, participants moved the stylus at lower speed at a smaller scale. In our experimental setting, the width value declined at the smaller scale, and thus the participants could not attain high speed. This supported the local speed form of the steering law [1], i.e., the current speed decreases with narrower path tolerance.

In the results of Yamanaka and Miyashita's study, the speeds of narrowing and widening changed over at $25 \%$ to $30 \%$ of the tunnel amplitude [24]. Similarly to their report, our results showed that narrowing was at a higher speed than widening until nearly $25 \%$ of each $A$ (Figure 7), because in the early phase the width was wider for narrowing than for widening. However, after the changeovers, widening retained higher speed than narrowing until the goal, and widening required significantly less movement time in total. Overall, except for scale effects, transitions and changeovers of cursor speed showed results similar to those in [24]. 


\section{Model Fitness of IDGap}

As mentioned in previous work [24], the steering law holds when the tunnel type is divided (top row of Figure 6). Therefore, we can predict the steering time at a high accuracy without the $I D_{\text {Gap }}$ model, if the $M T$ s for both types are measured sufficiently. Where the tunnel type is not separated, the conventional steering law shows a bad fit (middle row of Figure 6). In particular, the steering time difference tends to increase with increasing $I D$ (Figure 10). This means that by using the measured data at low $I D$ values, we predict a less accurate $M T$ at a high $I D$. If one would like to predict $M T$ values at higher IDs more easily, especially $M T$ values of narrowing tunnels, whose measurement is time-consuming, the $I D_{\text {Gap }}$ model helps to predict with high accuracy, as shown in the bottom row of Figure 6.

One of our goals was to investigate whether there is a steering time difference between narrowing and widening tunnels at any scale. In fact, the time difference appeared at all scales from $S=1$ to 12 (Figure 10), hence, we confirmed the necessity of a model for predicting $M T_{\mathrm{NT}}$ from $M T_{\mathrm{WT}}$. In our experiment, the $I D_{\text {Gap }}$ model showed a good fit at all scales; therefore, the adequateness of the model was confirmed at the scale range we used. Importantly, because the time difference tends to be larger with decreasing scale (Figure 10), the usefulness of the prediction model would increase at smaller scales; in addition to [24] we showed that the $I D_{\text {Gap }}$ model is valid even for scaled tunnels. However, the usefulness does not decrease at a large scale because the fit using the $I D_{\mathrm{Gap}}$ model improves compared with the conventional steering law. Therefore, the $I D_{\text {Gap }}$ model covers the scale that requires primarily finger movements to upper arm movements, and we confirm that there is wide applicability from the viewpoint of that scale.

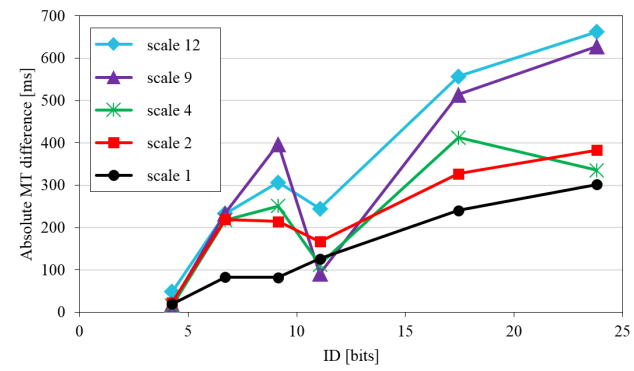

Figure 10. Difference between $M T_{\mathrm{NT}}$ and $M T_{\mathrm{WT}}$ at each scale.

\section{Experimental Design Separating the Motor and Visual Scales}

We used a direct-input pen tablet as the experimental device. This condition allowed us to investigate the scale effects for which both motor and visual scales changed together, a common environment for drawing. However, because we are also interested in the scale effects when the two scale types are divided, we discuss the experimental design for it.

First, to separate the motor and visual scales, an indirectinput pen tablet is required. In addition, there are two choices of position control methods, namely, absolute or relative. In the case of absolute control, the motor scale can be controlled by the CD gain. Accot and Zhai used a display and a stylus pad that had the same size and the same aspect ratio (24 inches, $1920 \times 1200$ pixels) [3]. The CD gain could be changed by configuring the active stylus pad area; if participants were required to use $1 / 2$ of the entire pad in the $\mathrm{x}$ - and $\mathrm{y}$-axis, the gain was set to 2 .

In the case of a different aspect ratio between the stylus pad and the display with absolute control, their form factors will affect the performance [12]. For example, for aspect ratios of the stylus pad and the display of $4: 3$ and 16:9, respectively, the slippage of the pen tip (input) in the y-axis is converted to be smaller on the display (output), and therefore, the error rates in horizontal tunnels might decrease. Conversely, in vertical tunnels, the slippage in the $\mathrm{x}$-axis becomes sensitive and will immediately cause an error.

In the case of relative control, such as a touchpad on a laptop PC, we must consider the effect of clutching (replacing the pen tip). The smaller the stylus pad, the more frequently clutching is required. Although the conventional steering law holds even if the clutching is executed using a touchpad and a trackball [2], the clutching frequency is a factor for the operation time in pointing tasks using a relative control stylus [6]. Taken together, conditions of direct/indirect input, absolute/relative control, aspect ratio difference, stylus pad size, and clutching frequency could affect performance, but our experiment included limited conditions on these considerations. To investigate the adequateness of the $I D_{\text {Gap }}$ model with separate motor and visual scales, the experimental conditions described above must be considered in a further study.

\section{Cursor Size}

We omitted another choice of parameter setting, i.e., visual size of the cursor. In our study, the crosshair cursor was 25 pixels $(6.19 \mathrm{~mm})$ in length at all scales. Of course, changing the cursor size depending on the $S$ conditions is a possibility. However, $1 / 12$ the size of 25 pixels $(=2$ or 3 pixels in length $<1 \mathrm{~mm}$ ) is too difficult to see, and we were concerned that participants would frequently miss the current cursor position.

Considering that our principal observation was the scale effect in the steering performance difference, we determined that disturbance of the steering performance by low visibility using an excessively small cursor must be avoided. Another reason was that if the $M T$ increased with decreasing scale, the experimental result would have a particular level of cursor visibility effect. One might think that this is also a scale effect, but in a pilot study by the authors, a cursor whose length was less than $1 \mathrm{~mm}$ was irritating and unsuitable for steering performance observation. Moreover, in many drawing tools and illustration applications, the cursor size is not changed dynamically by zooming its canvas. We thought that a fixed 
cursor size appeared to be natural for liquid crystal pen tablet operations.

\section{Free Weight $k$}

Another important point in question is the free weight $k$. Yamanaka and Miyashita considered that $k$ was affected by the number of movement corrections in a tunnel, the degree of change of $W$ and $A$, or the operation device [24]. In our study, $k$ ranged from 3 to 6 ; however, the relationship between these $k$ values and other results or conditions (such as $M T_{\mathrm{NT}}, M T_{\mathrm{WT}}$, scales, and tunnel parameters) is still uncertain. At least, there appears to be no optimal $k$ value for all scales.

It is not surprising that $k$ changes with changing device or tunnel shape, as suggested by Yamanaka and Miyashita [24]. However, in our study, $k$ was altered when only the scale was changed and the other conditions ( $W, A$, or device) were fixed. Therefore, this result shows a new limitation of the $I D_{\mathrm{Gap}}$ model; the optimal $k$ value must be calculated at each scale to predict $M T_{\mathrm{NT}}$ from $M T_{\mathrm{WT}}$.

\section{CONCLUSION AND FUTURE WORK}

In this study, we conducted an experiment that tested the scale effects in the steering time difference between narrowing and widening linear tunnels. The input and output device was a directly manipulated liquid crystal pen tablet, which simultaneously changed motor and visual scales. The results showed that the larger the scale, the smaller were the $M T$ values and errors. This contrasts with the results of Accot and Zhai's scale study [3]; in our study, the performance did not show a U-shaped function. There were steering time differences between the tunnel types at all scales tested ( $S=1$ to 12 ), and the $I D_{\text {Gap }}$ model showed good fit at all scales.

A limitation in previous work on narrowing and widening tunnels was that the fitness of the $I D_{\text {Gap }}$ model was confirmed at one scale [24], which required primarily wrist movements with a forearm extension. Our experimental results showed that the usefulness of the model was observed in a wider range of scales from finger movements to upper arm movements. However, our study was also conducted using only straight tunnels. Our next interest is the steering time difference between narrowing and widening curved tunnels, including not only circular tunnels but also unclosed loops. What happens to the steering performance when the narrowing and widening tunnels become similar to circular shapes, as shown in Figure 11? What happens when a tunnel has more than one loop? Is there always a time difference between the narrowing and widening directions? Does the $I D_{\mathrm{Gap}}$ model hold for any curvature factor? Further investigation is required to answer these questions.

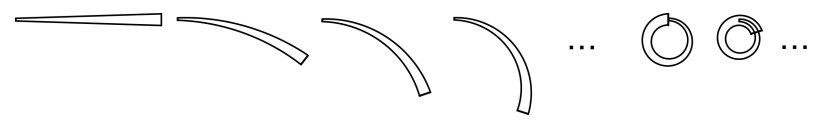

Figure 11. Width-changing tunnels with various curvature factors, all of which have the same $A$ and $W$.

\section{ACKNOWLEDGMENTS}

This work was supported by JSPS KAKENHI Grant Number 15J11634, and OngaCREST, JST.

\section{REFERENCES}

1. Accot, J. and Zhai, S. 1997. Beyond Fitts' law: models for trajectory-based HCI tasks. In Proceedings of the SIGCHI Conference on Human Factors in Computing Systems (CHI '97), 295-302.

http://dx.doi.org/10.1145/258549.258760

2. Accot, J. and Zhai, S. 1999. Performance evaluation of input devices in trajectory-based tasks: an application of the steering law. In Proceedings of the SIGCHI Conference on Human Factors in Computing Systems (CHI '99), pp.466-472.

http://dx.doi.org/10.1145/302979.303133

3. Accot, J. and Zhai, S. 2001. Scale effects in steering law tasks. In Proceedings of the SIGCHI Conference on Human Factors in Computing Systems (CHI '01), pp.1-8. http://dx.doi.org/10.1145/365024.365027

4. Browning, G. and Teather, R. J. 2014. Screen scaling: effects of screen scale on moving target selection. In Extended Abstracts of the SIGCHI Conference on Human Factors in Computing Systems (CHI '14), 2053-2058.

http://dx.doi.org/10.1145/2559206.2581227

5. Casiez, G., Plénacoste, P., and Chaillou, C. 2004. Does DOF separation on elastic devices improve user 3D steering task performance? In Proceedings of the Asia Pacific Conference on Computer Human Interaction (APCHI '04), pp.70-80. http://dx.doi.org/10.1007/978-3-540-27795-8 8

6. Casiez, G., Vogel, D., Pan, Q., and Chaillou, C. 2007. RubberEdge: reducing clutching by combining position and rate control with elastic feedback. In Proceedings of the ACM Symposium on User Interface Software and Technology (UIST '07), 129-138. http://dx.doi.org/10.1145/1294211.1294234

7. Chapuis, O. and Dragicevic, P. 2011. Effects of motor scale, visual scale, and quantization on small target acquisition difficulty. ACM Transactions on ComputerHuman Interaction (TOCHI), Vol.18, No.3, Article 13. http://dx.doi.org/10.1145/1993060.1993063

8. Dennerlein, J. T., Martin, D. B., and Hasser, C. 2000. Force-feedback improves performance for steering and combined steering-targeting tasks. In Proceedings of the SIGCHI Conference on Human Factors in Computing Systems (CHI '00), 423-429. http://dx.doi.org/10.1145/332040.332469

9. Fitts, P. M. 1954. The information capacity of the human motor system in controlling the amplitude of movement. Journal of Experimental Psychology, Vol.47, No.6, 381-391. http://psycnet.apa.org/doi/10.1037/h0055392 
10. Forlines, C. and Balakrishnan, R. 2008. Evaluating tactile feedback and direct vs. indirect stylus input in pointing and crossing selection tasks. In Proceedings of the SIGCHI Conference on Human Factors in Computing Systems (CHI '08), 1563-1572. http://dx.doi.org/10.1145/1357054.1357299

11. Gibbs, C. B. 1962. Controller design: interactions of controlling limbs, time-lags, and gains in positional and velocity systems. Ergonomics, Vol.5, No.2, 385-402. http://dx.doi.org/10.1080/00140136208930602

12. Gilliot, J., Casiez, G., and Roussel, N. 2014. Impact of form factors and input conditions on absolute indirecttouch pointing tasks. In Proceedings of the SIGCHI Conference on Human Factors in Computing Systems (CHI '14), 723-732. http://dx.doi.org/10.1145/2556288.2556997

13. Gutwin, C. and Skopik, A. 2003. Fisheyes are good for large steering tasks. In Proceedings of the SIGCHI Conference on Human Factors in Computing Systems (CHI '03), 201-208. http://dx.doi.org/10.1145/642611.642648

14. Hess, R. A. 1973. Nonadjectival rating scales in human response experiments. Human Factors, Vol.13, No.3, 275-280.

15. Jellinek, H. D. and Card, S. K. 1990. Powermice and user performance. In Proceedings of the SIGCHI Conference on Human Factors in Computing Systems (CHI '90), 213-220. http://dx.doi.org/10.1145/97243.97276

16. Kattinakere, R. S., Grossman, T., and Subramanian, S. 2007. Modeling steering within above-the-surface interaction layers. In Proceedings of the SIGCHI Conference on Human Factors in Computing Systems (CHI '07), 317-326. http://dx.doi.org/10.1145/1240624.1240678

17. Kulikov, S., MacKenzie, I. S., and Stuerzlinger, W. 2005. Measuring the effective parameters of steering motions. In Extended Abstracts of the SIGCHI Conference on Human Factors in Computing Systems (CHI '05), 1569-1572. http://dx.doi.org/10.1145/1056808.1056968

18. Kulikov, S. and Stuerzlinger, S. 2006. Targeted steering motions. In Extended Abstracts of the SIGCHI Conference on Human Factors in Computing Systems (CHI '06), 983-988.

http://dx.doi.org/10.1145/1125451.1125640

19. Liu, L., Martens, J.-B., and Liere, R. v. 2011. Revisiting path steering for $3 \mathrm{D}$ manipulation tasks.
International Journal of Human-Computer Studies, Vol.69, Issue 3, 170-181. http://dx.doi.org/10.1016/j.ijhcs.2010.11.006

20. Naito, S., Kitamura, Y., and Kishino, F. 2004. Steering law in an environment of spatially coupled style with matters of pointer size and trajectory width. In Proceedings of the Asia Pacific Conference on Computer Human Interaction (APCHI '04), 305-316. http://dx.doi.org/10.1007/978-3-540-27795-8_31

21. Pastel, R. 2006. Measuring the difficulty of steering through corners. In Proceedings of the SIGCHI Conference on Human Factors in Computing Systems (CHI '06), 1087-1096.

http://dx.doi.org/10.1145/1124772.1124934

22. Senanayake, R. and Goonetilleke, R. S. 2015. Setting that mouse for tracking tasks. In Proceedings of International Conference on Digital Human Modeling and Applications in Health, Safety, Ergonomics and Risk Management (DHM '15), Vol.2, 276-281. http://dx.doi.org/10.1007/978-3-642-39182-8_33

23. Sun, M., Ren, X., Zhai, S., and Mukai, T. 2012. An investigation of the relationship between texture and human performance in steering tasks. In Proceedings of the Asia Pacific Conference on Computer Human Interaction (APCHI '12), 1-6. http://dx.doi.org/10.1145/2350046.2350048

24. Yamanaka, S. and Miyashita, H. 2016. Modeling the steering time difference between narrowing and widening tunnels. In Proceedings of the SIGCHI Conference on Human Factors in Computing Systems (CHI '16), 1846-1856. http://dx.doi.org/10.1145/2858036.2858037

25. Yin, J., Ren, X., and Zhai, S. 2010. Pen pressure control in trajectory-based interaction. Behaviour \& Information Technology, Vol.29, No.2, 137-148. http://dx.doi.org/10.1080/01449290902904733

26. Zhou, X., Ren, X., and Hui, Y. 2008. Effect of start position on human performance in steering tasks. In Proceedings of the International Conference on Computer Science and Software Engineering (CSSE '08), Vol.2, 1098-1101. http://dx.doi.org/10.1109/CSSE.2008.1310

27. Zhai, S., Accot, J., and Woltjer, R. 2004. Human action laws in electronic virtual worlds: an empirical study of path steering performance in VR. Presence, Vol.13, No.2, 113-127. http://dx.doi.org/10.1162/1054746041382393 